\title{
Ni urbanos, ni rurales: cambios intergeneracionales en adscripción territorial subjetiva en la región del Maule, Chile
}

\section{Felipe Saravia}

Trabajador social, magíster en Desarrollo Local y Regional, candidato a doctor en Ciencias Sociales en Estudios Territoriales. Académico en Departamento de Ciencias Sociales, Escuela de Trabajo Social, Universidad del Bío-Bío, Chile. fsaravia@ubiobio.cl

\section{Francisco Letelier}

Sociólogo, magíster en sociología, doctorando en Geografía, Planificación Territorial y Medio Ambiente. Académico en Departamento de Ciencias Sociales, investigador en CEUT, Universidad Católica del Maule, Chile. fletelier@ucm.cl.

\section{Stefano Micheletti}

Titulado en Ciencias Forestales y Ambientales, magíster en cooperación internacional. Académico en Departamento de Ciencias Sociales, investigador en CEUT y CEDERE, Universidad Católica del Maule, Chile.smicheletti@ucm.cl.

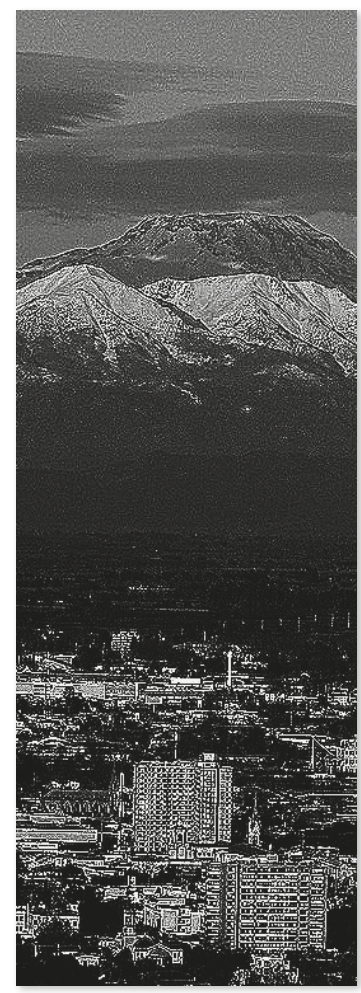




\title{
Resumen
}

Utilizando los datos de una encuesta aplicada a 1634 personas de la región del Maule de Chile en 2014, se examina la evolución intergeneracional de las percepciones subjetivas de adscripción territorial y su vínculo con definiciones objetivas de ruralidad y urbanidad. El estudio tiene un alcance correlacional y utiliza el estadístico Chi cuadrado para determinar asociaciones entre variables. Se corrobora la hipótesis: las adscripciones territoriales subjetivas no siempre coinciden con las tipificaciones territoriales administrativas, y los cambios de adscripción entre generaciones sucesivas no transitan necesariamente desde lo rural a lo urbano, sino que dan lugar a perfiles con características diversas, siendo uno de los más importantes el que se asienta en la hibridación urbano-rural. Se concluye que la región del Maule es cada vez más mixta en términos identitarios; así, se aporta evidencia concreta para el cuestionamiento a la idea de que el avance de lo urbano es lineal y unívoco.

\section{Palabras clave}

Urbanidad; urbanización; ruralidad; adscripción territorial subjetiva.

\begin{abstract}
Neither urban nor rural: intergenerational changes in subjective territorial affiliation in the Maule Region of Chile.

Using data from a 2014 survey of 1,634 people in the Maule region of Chile, we examine the intergenerational evolution of subjective perceptions of territorial affiliation and its link with objective definitions of rurality and urbanity. The study applies a correlational method the "chi-squared test" to determine the associations between variables. The hypothesis is corroborated: subjective territorial affiliations do not always coincide with administrative territorial classifications, and changes of affiliation between successive generations do not necessarily go from rural to urban, but give rise to profiles with diverse characteristics, one of the more important being a rural-urban hybridization. It is concluded that the Maule region is increasingly mixed in terms of identity, thus providing concrete evidence to challenge the idea that urban progress is linear and having only one meaning.
\end{abstract}

\section{Keywords}

Urbanity; urbanization; rurality; subjective territorial affiliation. 


\section{Introducción}

En el marco de lo que algunos autores como BRENNER y SCHMID (2016) denominan proceso de urbanización global, de las persistentes dicotomías que oponen lo rural a lo urbano y que además sostienen el tránsito sistemático desde la primera a la segunda condición, este trabajo examina el correlato entre las definiciones objetivas de ruralidad y urbanidad y las percepciones subjetivas de adscripción territorial. Se parte de las siguientes interrogantes: ¿en qué medida se asocia el habitar en un lugar definido oficialmente como urbano o rural con la adscripción territorial subjetiva de los sujetos que allí habitan?; dicho en otros términos: ¿se considera a sí mismo urbano quien vive en lo que denominamos ciudad o rural aquel que vive en lo que denominamos campo? y ¿qué características tienen los cambios intergeneracionales de dichas percepciones?

Se plantea como hipótesis que las autopercepciones de ruralidad o urbanidad de los sujetos no se asocian de forma lineal con las tipificaciones que se hacen desde las lógicas administrativas de los territorios en los que habitan, y que aunque en términos generales la transición histórica es desde lo rural a lo urbano, las múltiples formas y espacios en que se produce la ocupación del territorio en el modelo de producción capitalista rompen esta dirección histórica y fragmentan las trayectorias territoriales e identitarias de los sujetos. Lo anterior cuestiona las formas tradicionales de definir lo urbano y lo rural, por cuanto no estarían dando cuenta de las maneras de experimentar el vínculo con los territorios. En este sentido, el análisis que se desarrolla no solo busca caracterizar las dinámicas mencionadas, sino contribuir al debate general respecto de la definición de lo urbano y lo rural, y el cuestionamiento a la idea de que hoy imperaría una era urbana global y homogénea.

El caso analizado es el de la región del Maule, que se ubica en la zona central de Chile, aproximadamente a 200 kilómetros al sur de la capital nacional. De acuerdo con las estimaciones del Instituto Nacional de Estadísticas, alberga una población de un poco más de un millón de personas (INE, 2002 ${ }^{1}$ ). De acuerdo con las mediciones oficiales realizadas por la Encuesta de Caracterización Socioeconómica (CASEN), ha sido sostenidamente en el tiempo la región más rural de Chile. En efecto, como se observa en el gráfico 1, a pesar de que durante los últimos quince años se aprecia un descenso importante en la población rural en todo Chile, esta tendencia presenta una brecha importante entre los datos de la región del Maule y el promedio nacional, lo que otorga aún mayor pertinencia al análisis propuesto.
1. El censo aplicado el año 2012 tuvo serios problemas técnicos que imposibilitan su uso confiable, por lo que el de 2002 es usado hasta ahora como fuente de datos oficial. 


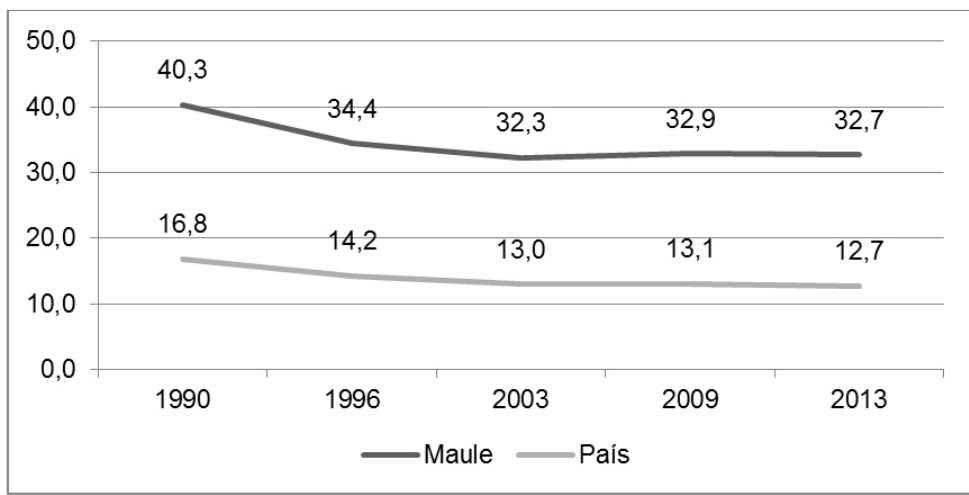

Figura 1. Evolución de la proporción de población rural del Maule y Chile Fuente: elaboración propia sobre la base de CASEN 1990, 1996, 2003, 2009 y 2013

El artículo se desarrolla siguiendo el siguiente esquema: en primer lugar, se presenta un marco referencial que critica la manera en que se definen y miden lo urbano y lo rural, y la idea de proceso mundial de urbanización. En segundo lugar, se describen brevemente algunas corrientes teóricas que proponen nuevas formas de entender la relación urbano-rural. En tercer lugar, se presentan el marco metodológico, los resultados obtenidos y la discusión de estos, y finalmente se concluye con algunas reflexiones a partir de los resultados y el planteamiento de nuevas interrogantes investigativas que surgen a la luz de estas.

\section{Antecedentes teóricos}

\section{La dicotomía rural-urbano y el proceso de urbanización}

Diversos autores han descrito las características propias de una ruralidad que se definía a partir de su intrínseca "no-urbanidad" (con los evidentes vacíos conceptuales que esto implicaba) y de su carácter agrario. La obra de TonNIEs (ÁlLVARo, 2010) abrió la puerta para que investigadores como NisBet (1967), Sorokin, Zimmermann y Galpin (1929) y posteriormente Germani (1963) y Solari (1968) se empeñaran -en contextos históricos distintos- en articular un relato basado en ideas fuerza contrapuestas que oponían comunidad y sociedad, tradición y modernidad, rural y urbano. Al definir lo rural como negación de lo urbano, los territorios rurales quedaron conceptualizados como regidos por fenómenos de carácter natural, con densidad poblacional baja y con componentes culturales tradicionales que enfatizaban la comunidad (Concha y otros, 2012). Por otro lado, lo urbano se asimiló directamente a la ciudad, la que quedó caracterizada por su gran tamaño y densidad poblacional, funcionalidad y complejidad en sus vínculos y, como resultado, por un modo de vida propio (WIRTH, 1938), que combinaba indiferencia y anonimato en el encuentro con los demás.

En el esquema anterior lo rural representa el extremo tradicional y atrasado, mientras 
lo urbano encarna la modernidad y la prosperidad. La noción de desarrollo se asociaba con la dirección de transformaciones unívocas y precisas que iban desde lo rural hacia lo urbano, es decir, de lo agrario a lo industrial. En el año 2007 dos agencias de la ONU, la United Nations Population Fund y ONU-Habitat, decretaban que la humanidad había entrado de lleno en la era urbana: más de la mitad de la población pasaba a vivir en ciudades (UNITED Nations Population Fund, 2007: 1 y ONU-Habitat, 2007: 1). Esta proporción iría creciendo para llegar al 75 \% en 2050 (BuRdetT y Rode, 2006: 8). La dualidad urbano/rural y, por lo tanto, la idea de un avance de la ciudad (tanto en términos geográficos como identitarios) sobre todo aquello que no lo es son generalizaciones que parecen ofrecer un "mapa cognitivo" viable (JAMEson, 1988) para navegar por un paisaje socio-espacial de rápida reestructuración en todo el mundo, definido este por la turbulencia, la incertidumbre y las descontroladas tendencias de crisis, a la vez sociales, espaciales y ambientales (BrenNer y Schmid, 2016).

Hoy, sin embargo, entendemos que los conceptos rural y urbano se refieren a condiciones extremadamente heterogéneas dentro y entre los territorios nacionales. "En su mayor parte la noción de lo rural utilizada en el discurso de la era urbana es simplemente una 'caja negra': se refiere a las zonas residuales de asentamiento que son supuestamente 'no urbanas', pero esto se hace sin especificar cuáles son las características que estos espacios pueden compartir en distintos contextos, ya sea en términos de tamaño de la población, densidad o composición, uso del suelo, mercados de trabajo u otros indicadores” (BRENNER y SCHMid, 2016: 329). Lo mismo ocurre con lo urbano: se pretende generalizar la idea de un avance a la condición urbana, mientras existe empíricamente una multiplicidad de condiciones de asentamientos que van desde la ciudad pequeña a la metrópolis (Brenner y Schmid, 2016). Se requieren, por tanto, nuevas conceptualizaciones que permitan repensar la forma en que describimos los diversos territorios.

\section{Nuevas formas de entender lo urbano-rural}

A partir de los años 70, las realidades latinoamericanas se han visto tensionadas por una serie de transformaciones sociales y productivas resultantes del despliegue del capitalismo neoliberal, y en especial del proyecto agroindustrial, que ha generado una serie de flujos e intercambios nuevos entre lo rural y lo urbano. En este contexto - aunque con algo de atraso- el debate teórico ha ido transitando hacia enfoques más integradores e integrales, con mayor capacidad de interpretar las novedosas dinámicas sociales y económicas en acto. En Chile, uno de los primeros intentos conceptuales fue la "Nueva Ruralidad” (PÉrEz, 2001; Gó- 
MEz, 2002 y 2003), que propuso redefinir lo rural a partir de tres dimensiones acumulativas: su multifuncionalidad en contextos de baja densidad demográfica, la especificidad derivada de relaciones vecinales prolongadas y la existencia de intensas relaciones de parentesco entre una parte significativa de los habitantes, y finalmente el mayor alcance territorial que abarca lo rural. En este sentido, la condición rural se hace coincidente con la de "provinciano”, más que con una actividad productiva específica.

Si bien este enfoque no permitió interpretar cabalmente las transformaciones en curso (en efecto, en lugar de un nuevo habitante rural, se estaba desarrollando en Chile una nueva agricultura, basada en un proyecto agroindustrial y de exportación), al menos permitió mover de las posiciones dicotómicas el foco del debate, a la vez que insinuar una primera compenetración rural-urbana a partir de la especificidad de las relaciones sociales de ciertos territorios intermedios. Desde de este primer impulso, la relación del "campo" con los pueblos y ciudades cercanas fue incorporada como elemento central en el análisis, buscando explicar las relaciones dinamizadoras de los polos que constituyen las urbes intermedias agrarias.

BERDEGUÉ Y OTROS (2010) plantearon una investigación interesante en este sentido, identificando la existencia de "ciudades rurales", que se ubican en un punto intermedio de un gradiente de ruralidad que va desde un extremo urbano (metrópolis) hasta uno rural (caseríos). Las ciudades rurales corresponderían a asentamientos que, si bien bajo el criterio de tamaño poblacional deberían ser considerados como urbanos, mantienen en la práctica una pauta de relaciones sociales que asociamos más a la realidad rural, por tener una vinculación orgánica y funcional con las actividades económicas agrícolas.

CANALES y HeRnÁNDEZ (2011) ponen en duda la compatibilidad de las categorías rural y urbana para la definición de un mismo territorio, y proponen el concepto de "agrópolis". Se atiende en este caso a la función productiva del territorio, haciendo una distinción entre "rural" y "agrario" y desplazando la tradicional dicotomía urbano/rural hacia el clivaje metrópolis/ agrópolis. La agrópolis se entiende en este caso como una red de poblamientos que se distribuyen en uno o varios valles, ciudades y pueblos, con actividades económicas piscisilvoagropecuarias y pluri-centradas.

Si bien estos enfoques abren el debate al salir de la clasificación dual de los territorios, se centran más bien en los aspectos económico-productivos, y abordan solamente de forma 
tangencial una cuestión central como la identidad. En este sentido, una aproximación micro-sociológica es la que proponen diversos autores como Kenbel (2006), GalimberTi (2011), Cimadevilla (2014) y Micheletti y Letelier (2016), quienes debaten la cuestión rural-urbana desde una perspectiva socio-productiva. Se habla entonces de ruralización de lo urbano, entendiéndose con ello el proceso en que lo urbano y lo rural se entremezclan y en su síntesis postulan lo rurbano (GAlimberti, 2011). Para SALCEDo y otros (2012), la categoría rurbana se manifiesta en la condición de vida de algunos actores cuyos modos y lógicas de acción basados en valores, saberes y sentires rurales comienzan a mimetizarse con la urbe, y viceversa. Podría pensarse en una relación dialéctica en que la cultura urbana se reapropia de bienes naturales y culturales habitualmente entendidos como propios del mundo rural, reforzando así los vínculos sociales.

Para Cimadevilla (2014), así como se ha pensado históricamente la urbanización de la vida rural, puede concebirse el proceso contrario, es decir, la ruralización de lo urbano, sin que por eso se extingan las situaciones precedentes. Se trataría de un proceso de interpenetración de los contrarios (Galimberti, 2011), que en la urbe se desarrolla en la medida en que "otros modos, estilos y lógicas de reproducción no siguen los parámetros de la razón dominante y se encarnan en los actores rurales que viven y/o trabajan en la ciudad" (KeNbel y Cimadevilla, 2009). Esto, a través de un conjunto de prácticas "rurbanas" que han sido catalogadas según su función productiva, laboral, comercial, de uso del tiempo libre y de residencia y sociabilidad.

Con todo, no queda resuelto el asunto ligado a la identidad de los sujetos y las comunidades. En este sentido - y a partir del análisis de un territorio específico-, el estudio "Identidad e Identidades del Maule" de la Universidad Católica del Maule y la ONG Surmaule (CoNCHA Y LETELIER, 2009) proponen una lectura interesante al constatar en términos prácticos el surgimiento de una categoría identitaria mixta ligada al proceso de hibridación de los territorios impactados por el proyecto agroindustrial en la región del Maule. Lo "rurbano" puede asociarse entonces al "acceso y al vínculo; a la libertad y a la comunidad; al anonimato y a la confianza; al pasado y al futuro, todo al mismo tiempo" (Concha y LeTelier, 2009). Esto significa la posibilidad de desvincularse de la tensión modernidad/tradición, y redefinir lo moderno "como aquella forma de habitar el territorio que promueve la integración de valores y ventajas; lo atrasado sería lo que tiende a aislar estos valores, oponerlos o intenta anular unos en privilegio de otros" (ConCHA y Letelier, 2009). 
Esta última propuesta nos permite de cierto modo cerrar el círculo de esta transición conceptual que iniciamos al mencionar los enfoques dicotómicos que asociaban una modernidad tecnológica e impersonal exclusivamente con lo urbano. Hoy es posible reconocer que el surgimiento de espacios e identidades híbridos permiten la construcción de una "nueva modernidad" a escala humana. En palabras de Touraine (BARBERo, s/f), "la idea del paso lineal de las tradiciones a la modernidad es sustituida por la afirmación de que la modernidad se define por la diversidad y multiplicación de las alternativas, la capacidad de asociar pasado y porvenir. Hay un cambio total de perspectiva: se consideraba que el mundo moderno estaba unificado mientras la sociedad tradicional estaba fragmentada; hoy por el contrario la modernización parece llevarnos de lo homogéneo a lo heterogéneo".

\section{Marco metodológico}

El artículo utiliza los datos de una encuesta aplicada cara a cara durante el año 2014 a 1634 personas mayores de 18 años residentes en la región del Maule, considerando cuatro submuestras, que corresponden al mismo tiempo a cuatro distintas escalas territoriales:

a) la zona urbana de la comuna de Talca, que es capital regional, con una población cercana a los 250.000 habitantes;

b) las zonas urbanas de las comunas de Curicó y Linares, capitales de las provincias de Curicó y Linares correspondientemente, con poblaciones entre los 100.000 y los 150.000 habitantes;

c) el conjunto de comunas con más de 15.000 habitantes y menos de 100.000 y

d) el conjunto de comunas con menos de 15.000 habitantes.

El muestreo fue aleatorio y tiene un nivel de confianza de $99 \%$ a nivel regional. Considerando un nivel de confianza de $95 \%$ a nivel de territorios subregionales, contempla un error inferior a $5 \%$ en el caso de Talca y las comunas con menos de 15.000 habitantes. En los otros dos territorios, el error muestral sobrepasa levemente el $5 \%$, lo que debe ser tomado en cuenta en las interpretaciones del análisis comparativo entre territorios. Es decir, se trata de una encuesta que se aproxima a algunos de los territorios definidos con un error muestral levemente más alto del estándar; sin embargo, permite de todas maneras un análisis aceptable respecto de los fenómenos en análisis en este artículo. 
Ni urbanos, ni rurales: cambios intergeneracionales en adscripción territorial subjetiva en la región del Maule, Chile

\begin{tabular}{|c|c|c|c|c|}
\hline \multicolumn{5}{|c|}{ Población regional estimada para 2014 y muestra de encuesta } \\
\hline & \multicolumn{2}{|c|}{ Población 2014} & \multicolumn{2}{|c|}{ Muestra encuesta } \\
\hline Territorio & $\mathrm{N}$ & $\%$ & $\mathrm{~N}$ & $\%$ \\
\hline Talca & 231.860 & $22 \%$ & 463 & $28 \%$ \\
\hline Curicó/Linares & 233.507 & $23 \%$ & 361 & $22 \%$ \\
\hline Más de 15 mil habitantes & 450.584 & $44 \%$ & 363 & $22 \%$ \\
\hline Menos de 15 mil habitantes & 119.642 & $12 \%$ & 447 & $27 \%$ \\
\hline Total & 1.035 .593 & $100 \%$ & 1.634 & $100 \%$ \\
\hline
\end{tabular}

Esta encuesta fue diseñada por el Centro de Estudios Urbanos y Territoriales de la Universidad Católica del Maule, y considera preguntas sobre percepción del territorio y sobre prácticas cotidianas y de participación social. Para efectos del presente artículo, se analizan las variables indicadas en el cuadro 2.

\begin{tabular}{|c|c|c|c|}
\hline \multicolumn{4}{|c|}{ Variables consideradas en el análisis } \\
\hline Variable & Pregunta & Respuestas posibles & $\mathrm{n}$ \\
\hline \multirow{5}{*}{$\begin{array}{l}\text { Territorio de } \\
\text { residencia }\end{array}$} & \multirow{5}{*}{$\begin{array}{l}\text { ¿En qué comuna } \\
\text { reside usted } \\
\text { actualmente la } \\
\text { mayor parte de } \\
\text { la semana? }\end{array}$} & a) Talca & 463 \\
\hline & & b) Curicó/Linares & 361 \\
\hline & & c) Núcleos urbanos (> de 15 mil habitantes) & 363 \\
\hline & & d) Sector Urbano/Rural (< de 15 mil Habitantes) & 447 \\
\hline & & Total & 1.634 \\
\hline \multirow{4}{*}{ Edad } & \multirow{4}{*}{$\begin{array}{l}\text { Edad (último } \\
\text { cumpleaños) }\end{array}$} & a) $18-29$ & 407 \\
\hline & & b) $30-59$ & 869 \\
\hline & & c) 60 o más & 359 \\
\hline & & Total & 1.635 \\
\hline \multirow{4}{*}{$\begin{array}{l}\text { Percepción } \\
\text { de ruralidad } \\
\text { o urbanidad } \\
\text { del padre }\end{array}$} & \multirow{4}{*}{$\begin{array}{l}\text { Pensando en su } \\
\text { padre, ¿usted diría } \\
\text { que él es o era una } \\
\text { persona...? }\end{array}$} & a) Totalmente rural & 319 \\
\hline & & b) Totalmente urbana & 615 \\
\hline & & c) Un poco rural y un poco urbana & 698 \\
\hline & & Total & 1.632 \\
\hline \multirow{4}{*}{$\begin{array}{l}\text { Autoper- } \\
\text { cepción de } \\
\text { ruralidad o } \\
\text { urbanidad }\end{array}$} & \multirow{4}{*}{$\begin{array}{l}\text { Pensando en } \\
\text { su vida, ¿usted } \\
\text { siente que es una } \\
\text { persona...? }\end{array}$} & a) Totalmente rural & 806 \\
\hline & & b) Totalmente urbana & 467 \\
\hline & & c) Un poco rural y un poco urbana & 320 \\
\hline & & Total & 1.593 \\
\hline
\end{tabular}

Fuente: elaboración propia 
El análisis consistió en estadística descriptiva de corte transversal, generando tablas de contingencia y aplicando también el estadígrafo Chi cuadrado. Los cruces de variables realizados se llevaron a cabo en dos fases. En primer lugar, se cruzó la variable autopercepción de ruralidad o urbanidad con el territorio de residencia, la edad y percepción de ruralidad o urbanidad del padre. En un segundo momento de análisis, se generó una tipología de los cambios y continuidades intergeneracionales en cuanto a adscripción territorial subjetiva, considerando cuatro categorías construidas a partir del cruce entre las variables autopercepción de ruralidad o urbanidad y percepción de ruralidad o urbanidad del padre. Se detalla la lógica utilizada en el cuadro 3.

\section{Cuadro 3}

Tipología de cambios y continuidades inter-generacionales en adscripción territorial subjetiva

\begin{tabular}{llcc}
\hline \multicolumn{1}{c}{ Tipo } & Detalle de cálculo & $\mathrm{n}$ \\
\hline $\begin{array}{l}\text { Mantención } \\
\text { rural }\end{array}$ & $\begin{array}{l}\text { Tienen padres considerados totalmente rurales y } \\
\text { que se consideran a sí mismas totalmente rurales }\end{array}$ & 284 & $22 \%$ \\
\hline Exrurales & $\begin{array}{l}\text { Tienen padres considerados totalmente rurales y que } \\
\text { no se consideran a sí mismas totalmente rurales }\end{array}$ & 520 & $41 \%$ \\
\hline $\begin{array}{l}\text { Mantención } \\
\text { urbana }\end{array}$ & $\begin{array}{l}\text { Tienen padres considerados totalmente urbanos y } \\
\text { que se consideran a sí mismas totalmente urbanas }\end{array}$ & 340 & $27 \%$ \\
\hline Exurbanos & $\begin{array}{l}\text { Tienen padres considerados totalmente urbanos y } \\
\text { que no se consideran a sí mismas totalmente urbanas }\end{array}$ & 127 & $10 \%$ \\
\hline Total & & $\mathbf{1 . 2 7 2}$ & $\mathbf{1 0 0 \%}$ \\
\hline
\end{tabular}

Fuente: elaboración propia

${ }^{i}$ El total de casos de la tabla 3 no coincide con el de la tabla 2, ya que en las tipologías de la tabla 3 no fueron consideradas las personas que tenían padres con identidades territoriales mixtas, con el fin de resaltar el contraste entre los cambios intergeneracionales de quienes tenían padres urbanos y rurales.

Esta tipología se cruzó con las variables territorio de residencia y sexo, aplicando también el estadígrafo Chi cuadrado. 
Ni urbanos, ni rurales: cambios intergeneracionales en adscripción territorial subjetiva en la región del Maule, Chile

\section{Resultados}

\section{Desacoples entre subjetividades y definiciones oficiales de ruralidad y urbanidad}

Un primer elemento asociado a la adscripción territorial subjetiva es el tipo de territorio de residencia. Al cruzar ambas variables aplicando Chi cuadrado se obtiene un $\mathrm{p}$ valor de 0,00 . La figura 2 da cuenta de las diferencias encontradas. Solo el $19 \%$ de la población regional dice sentirse totalmente rural. Ello contrasta con los niveles de ruralidad medidos por CASEN, como se observa en la figura 1. La mayor proporción se declara un poco rural y un poco urbana. Ello es así a nivel regional, y en las comunas con menos y más de 15.000 habitantes, exceptuando Curicó/Linares y Talca, que presentan las más altas proporciones de población que se percibe totalmente urbana. En este sentido, se observa que los territorios de mayor ruralidad definida según parámetros oficiales son aquellos que a su vez presentan las menores proporciones de personas que se perciben como totalmente urbanas y, consecuentemente, las más altas proporciones de personas que se perciben como totalmente rurales.

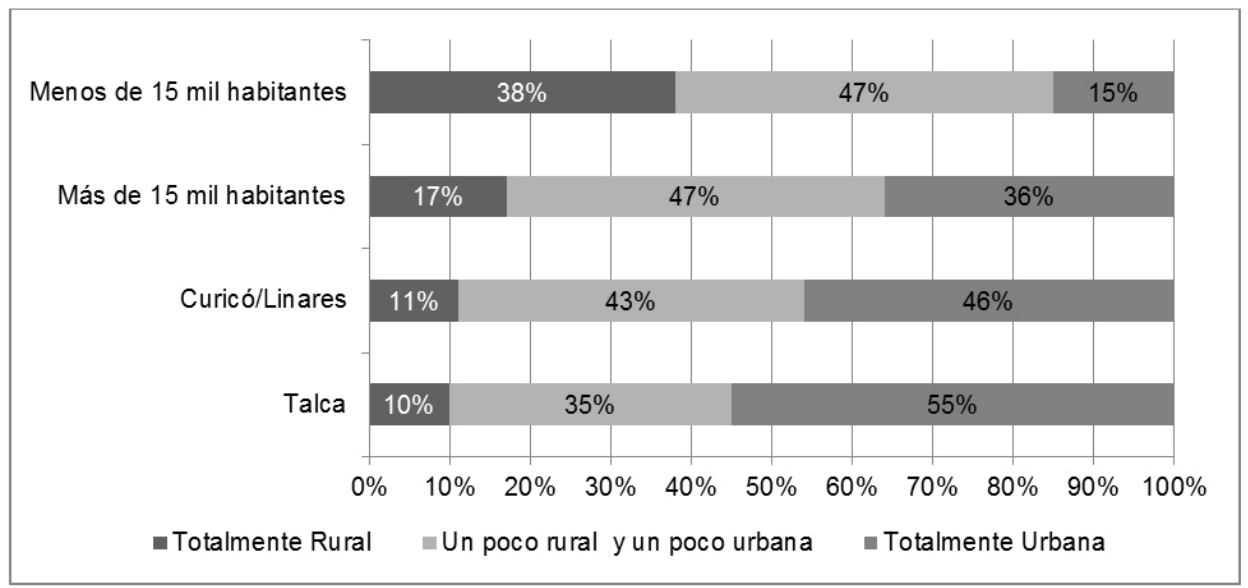

Figura 2. Adscripción territorial subjetiva según territorio de residencia Fuente: elaboración propia sobre la base de encuesta CEUT (2014) 
Pese a lo anterior, se observan algunos desacoples entre las definiciones oficiales de urbanidad/ruralidad y las autopercepciones de sus habitantes, lo que se refleja en que aún en las zonas urbanas de las ciudades intermedias hay un $10 \%$ de población que se percibe a sí misma como totalmente rural, y que, en el sentido opuesto, parte importante de personas que viven en territorios predominantemente rurales se sienten totalmente urbanas (15\% en ciudades de menos de 15.000 habitantes y $36 \%$ en las con menos de 100.000). De hecho, el $21 \%$ de quienes se declaran totalmente rurales viven en los cascos urbanos de ciudades intermedias, y el $32 \%$ de quienes se declaran totalmente urbanos viven en comunas que no son ciudades intermedias. Estas constataciones dan cuenta de maneras no lineales de experimentar el territorio, y ponen en cuestión las formas oficiales de definición de urbanidad y ruralidad.

\section{Argumentos para una crítica a la visión lineal de la urbanización}

Otro aspecto asociado a la autopercepción de ruralidad o urbanidad es la edad de la población ( $\mathrm{p}$ valor de 0,00 ). Como se observa en la figura 3, los adultos mayores (personas de 60 o más años) se declaran, en mayor medida que los otros rangos etarios, totalmente rurales. En contraposición sería esperable que los jóvenes (personas entre 18 y 29 años) fueran el rango etario que presenta la más alta proporción de personas que se declaran totalmente urbanas, pero no es así. Entre los jóvenes se observa la más alta proporción de personas que se perciben un poco rurales y un poco urbanos, es decir, con autopercepciones mixtas.

Como se mencionó anteriormente, la categoría un poco rural y un poco urbana es mayoritaria en la región en términos generales; sin embargo, cuando se compara entre distintos rangos etarios, se observa que esto es así en jóvenes y adultos, pero no en adultos mayores, lo que podría ser interpretado como que la experiencia que tuvieron los estratos adultos y jóvenes corresponde a una más rurbana, mientras que los adultos mayores fueron socializados en una época en la que las distinciones eran más claras. Este hecho podría también estar asociado a que los adultos mayores han vivido sus etapas vitales previas en períodos históricos en que la región tenía un nivel de urbanización menor, lo que incide en su autopercepción. Por otro lado, se descarta que esta diferencia se deba a la existencia de una población más envejecida en territorios definidos como más rurales, ya que las diferencias en este sentido no son estadísticamente significativas según la encuesta analizada, lo que se condice con los datos de la encuesta CASEN 2015. 
Ni urbanos, ni rurales: cambios intergeneracionales en adscripción territorial subjetiva en la región del Maule, Chile

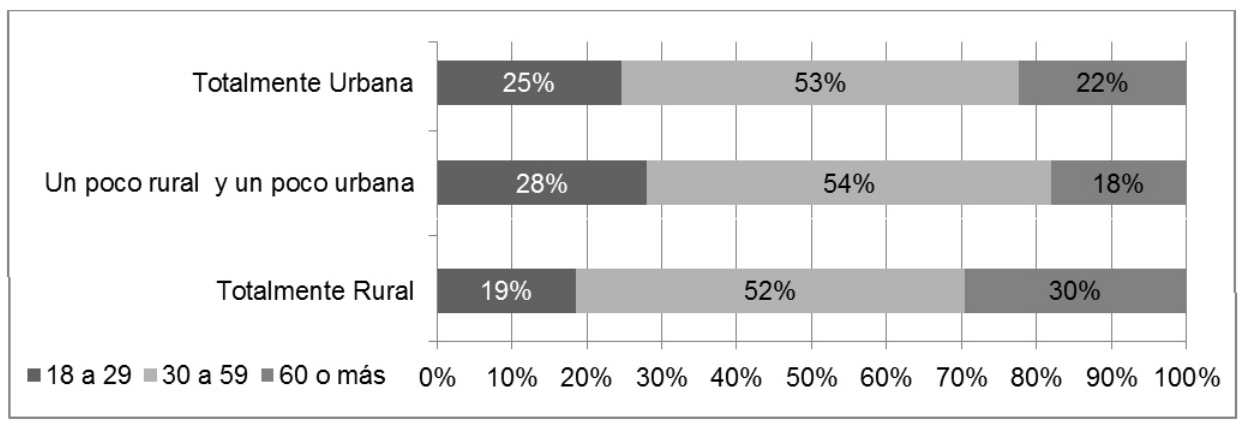

Figura 3. Adscripción territorial subjetiva según rango etario Fuente: elaboración propia sobre la base de encuesta CEUT (2014)

Para ahondar en los cambios intergeneracionales descritos en la figura 4, se cruza la variable autopercepción de ruralidad o urbanidad con la percepción que los encuestados tienen de la situación de ruralidad o urbanidad de sus padres. Se encontró que ambas variables se encuentran asociadas ( $p$ valor 0,00). Destaca el hecho de que el $46 \%$ de la población regional no se percibe de la misma manera que percibe a su padre, lo que da cuenta de importantes cambios intergeneracionales. Estos se han producido con más intensidad entre quienes tienen adscripciones territoriales subjetivas mixtas, ya que solo el $35 \%$ de ellos tenían padres considerados de la misma manera. En cambio, entre quienes se perciben totalmente urbanos, un 57 \% reproduce la adscripción territorial subjetiva de su padre, proporción que llega al 90 \% entre quienes se perciben totalmente rurales. Es decir, la dirección de los cambios intergeneracionales apunta principalmente hacia adscripciones territoriales subjetivas mixtas y en segundo lugar, a aquellas totalmente urbanas.

Estos hallazgos constituyen elementos importantes a partir de los cuales cuestionar la unidireccionalidad de los procesos de urbanización y la forma en que se define operacionalmente lo urbano y lo rural. Se está frente a un fenómeno de direccionalidades múltiples: por un lado, hay quienes teniendo padres totalmente rurales transitan hacia autopercepciones más urbanas, y quienes teniendo padres totalmente urbanos transitan hacia autopercepciones más rurales; cambios intergeneracionales que se producen predominantemente mediante el acrecentamiento de la categoría de percepción mixta, "un poco rural y un poco urbana", más que un tránsito directo desde una percepción rural a una urbana o viceversa. 


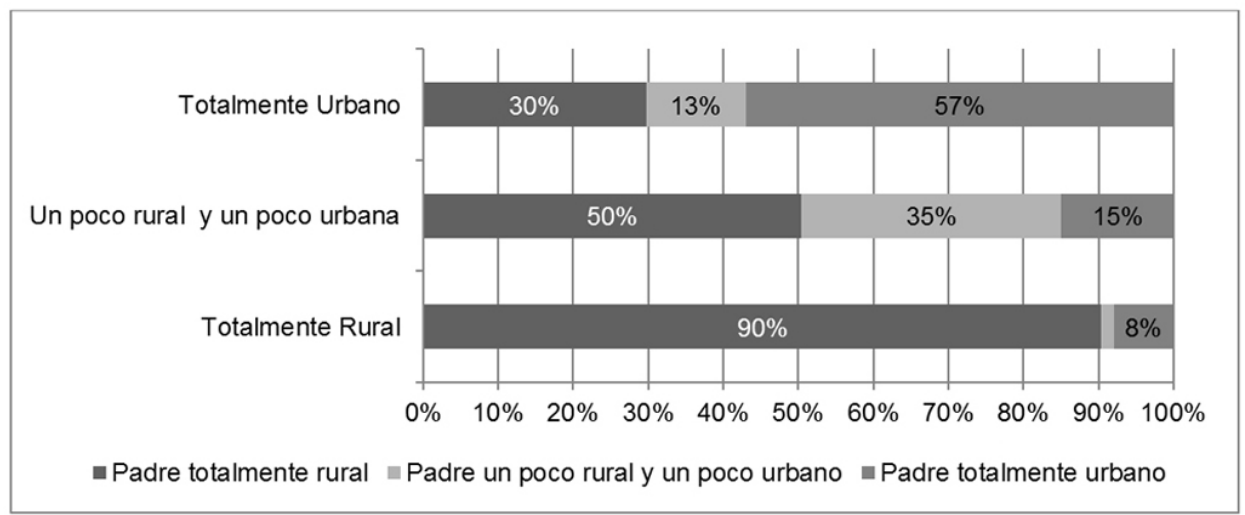

Figura 4. Cambios intergeneracionales en adscripción territorial subjetiva Fuente: elaboración propia sobre la base de encuesta CEUT (2014)

\section{Exploración preliminar del perfil de quienes cambian o reproducen intergeneracionalmente adscripciones territoriales subjetivas}

Como se detalló en la metodología, se construyó una tipología de cambios y continuidades intergeneracionales en adscripción territorial subjetiva (cuadro 3) cruzando las variables autopercepción de ruralidad o urbanidad y percepción de ruralidad o urbanidad del padre. Interesa ahondar en las características específicas de los distintos grupos identificados.

Existe una asociación entre los perfiles identificados y el territorio de residencia ( $p$ valor de 0,00 ). En la figura 5 se observa que las ciudades intermedias presentan la más alta proporción de permanencia intergeneracional urbana: 39 \% en Talca y 31 \% en Curicó/Linares, que disminuye en comunas menos pobladas, especialmente en aquellas de menos de 15.000 habitantes. En la permanencia intergeneracional rural se observa la dinámica inversa: se encuentran en esta categoría el $52 \%$ de los habitantes de comunas de menos de 15.000 habitantes, el 23 $\%$ en aquellas de más de 15.000 habitantes y llega a su más baja proporción en las ciudades intermedias. En suma, ambos tipos de reproducción intergeneracional de adscripciones territoriales subjetivas corresponden al $49 \%$ de los sujetos en la tipología generada. 
Quienes experimentaron cambio intergeneracional representan el $51 \%$ del total de la tipología, predominando el perfil exrurales, aunque los exurbanos representan el 19,6 \% del total de sujetos que experimentaron cambio intergeneracional. Esto resalta el hecho de que el proceso de urbanización no es lineal, dada la existencia de una parte importante de la población que genera tránsitos en sentido inverso al esperado.

Se observan diferencias en la distribución territorial de ambos perfiles de cambio intergeneracional, que son más pronunciadas en el caso de los exrurales. Este perfil representa el $34 \%$ de los habitantes de las comunas de más de 15.000 habitantes, el $25 \%$ en las comunas de menos de 15.000 habitantes y encuentra sus más bajas proporciones en las ciudades intermedias. Esto indica que es en los territorios menos poblados donde se ha vivenciado más fuertemente el alejamiento intergeneracional respecto de la adscripción rural. En el caso de los exurbanos, en cambio, estas diferencias no son tan pronunciadas, aunque también se observa que las comunas menos pobladas tienen entre tres y siete puntos porcentuales más que las ciudades intermedias. Es decir, el fenómeno de alejarse de adscripciones urbanas de la generación pasada tiene una distribución más homogénea en términos territoriales que el fenómeno del alejamiento de adscripciones rurales, aunque este último es más numeroso.

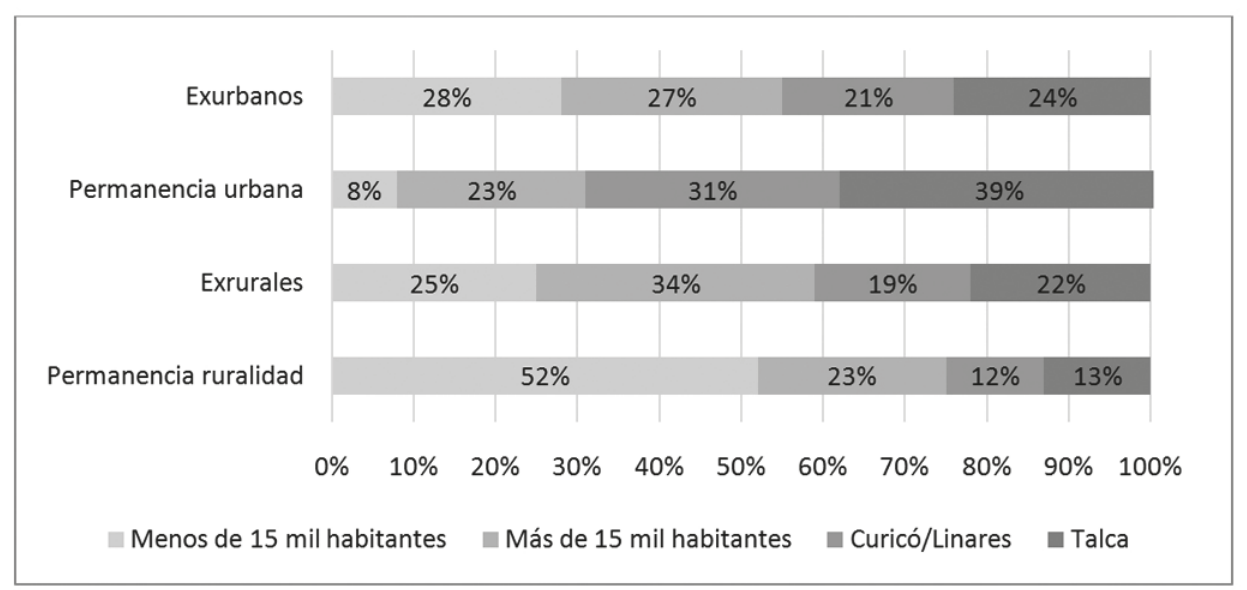

Figura 5. Territorios de perfiles según adscripciones territoriales subjetivas intergeneracionales Fuente: elaboración propia sobre la base de encuesta CEUT (2014) 
La tipología generada se encuentra asociada también a la edad de los sujetos ( $\mathrm{p}$ valor de $0,003)$. Se diferencian del promedio de edades del total de la muestra los perfiles de mantención rural, mantención urbana y exurbanos. En el perfil de mantención rural, los adultos mayores representan el $29 \%$, ubicándose así seis puntos porcentuales por sobre el promedio de la muestra en esta categoría, en contraste con lo que ocurre con el mismo rango etario del perfil de mantención urbana, donde representa el $17 \%$, seis puntos porcentuales por bajo el promedio de la muestra. Es decir, los adultos mayores se encuentran representados en mayor medida en el perfil mantención rural que en el perfil mantención urbana.

Por otro lado, el caso del perfil de exurbanos es distinto de los demás, ya que junto con concentrar la proporción más baja de adultos mayores de la muestra, es el único perfil en el que los adultos aparecen representados con el $62 \%$, siete puntos porcentuales por sobre la media de la muestra en esta categoría. Es decir, la experiencia de alejamiento de adscripciones territoriales subjetivas urbanas de la generación pasada es menos común entre adultos mayores y más común entre adultos. Ahora bien, los adultos exurbanos tienen una edad mediana de 42 años, la más baja de todos los otros perfiles, que llegan a tener una mediana de 48 años en el caso de los adultos de mantención rural.

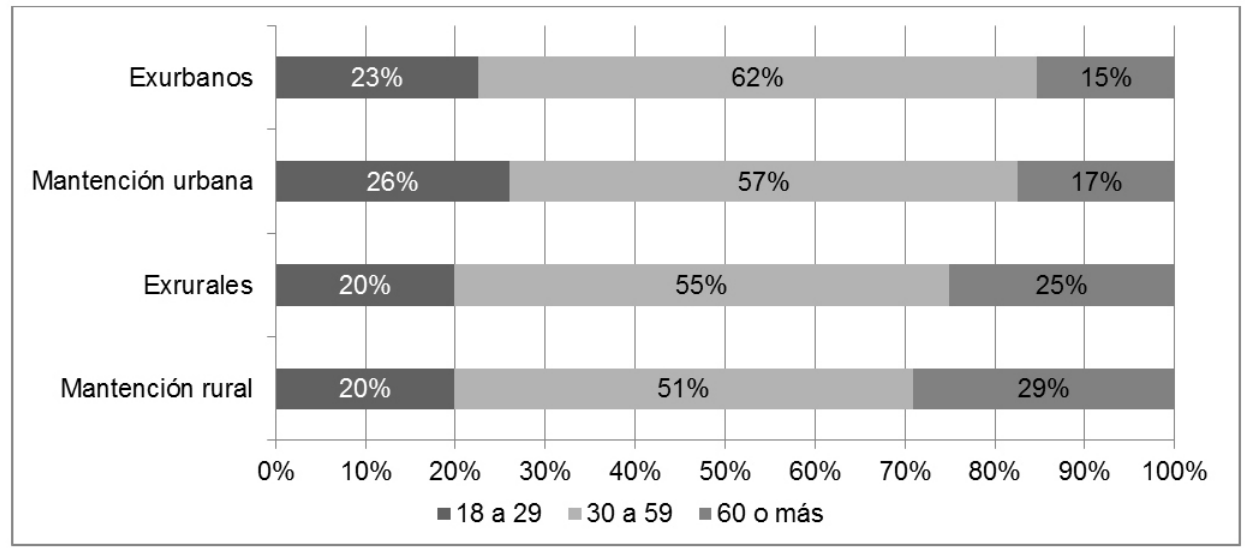

Figura 6. Edad de perfiles según adscripciones territoriales subjetivas intergeneracionales Fuente: elaboración propia sobre la base de encuesta CEUT (2014) 
En suma, se encuentra que cada perfil tiene características particulares que los distinguen del resto. El perfil de exrurales es más habitual en comunas de más de 15.000 habitantes y tiene una distribución según rango etario similar a la distribución de la muestra en su conjunto. Por su parte, el perfil de exurbanos se caracteriza por una proporción mayor a la media regional de adultos, y una proporción menor a la media regional de adultos mayores, y tiene una distribución territorial similar a la de la muestra en su conjunto. El perfil de mantención rural es más común entre adultos mayores de comunas con menos de 15.000 habitantes, en contraste con el perfil de mantención urbana, que es más común en las ciudades intermedias y tiene una proporción más baja de adultos mayores que la muestra en su conjunto.

\section{Reflexiones finales}

Los resultados del análisis constituyen fundamentos para aseverar la existencia de desacoples entre las definiciones oficiales de ruralidad y urbanidad con las adscripciones territoriales subjetivas. En los territorios oficialmente considerados urbanos habitan quienes se consideran a sí mismos rurales, y en los territorios oficialmente considerados rurales habitan quienes se consideran urbanos. Además, se encuentran extendidas adscripciones subjetivas mixtas que dan cuenta de formas complejas de vivenciar el territorio, que dificultan la separación taxativa entre lo urbano y lo rural.

Más específicamente, y a modo de síntesis, creemos que son muy importantes de relevar los siguientes puntos: en primer lugar, las divisiones territoriales administrativas no dan cuenta de los territorios funcionales y de los territorios simbólicos/identitarios. Esto no significa solamente que personas que se sienten urbanas vivan en zonas definidas como rurales por la institucionalidad, y viceversa (hay aquí un componente ligado a la trayectoria personal socio-espacial que permite una construcción identitaria individual); significa también que las lecturas que los sujetos hacen de su entorno difieren de las normativas: es posible encontrar ruralidad en lo urbano y urbanidad en lo rural. Esto implica que las prácticas socio-productivas cotidianas le otorgan un carácter al territorio (una identidad colectiva), y que posiblemente esto refuerza las autopercepciones mixtas. Aquí el discurso sobre la urbanización del campo - alimentado por la llegada del Estado y del despliegue del proyecto agroindustrial — es bastante difuso; en cambio, se está empezando a visibilizar el fenómeno de la ruralización de las ciudades. Con todo, hay un elemento central que CanaLEs y HeRnÁndez 
(2011) no advirtieron en sus investigaciones sobre el agrópolis: los territorios que no se ven no se gobiernan. Esto vale no solamente por su dimensión funcional, sino también por la simbólica/identitaria. En este sentido, el relevamiento de estas adscripciones territoriales subjetivas mixtas constituye un aporte importante.

Segundo, el estudio muestra que, por un lado, hay quienes teniendo padres totalmente rurales transitan hacia autopercepciones más urbanas, y quienes teniendo padres totalmente urbanos transitan hacia autopercepciones más rurales; cambios intergeneracionales que se producen predominantemente mediante el acrecentamiento de la categoría de percepción mixta "un poco rural y un poco urbana". Esto puede significar que está en construcción una síntesis identitaria interesante (o bien que esta síntesis está consolidada, y simplemente no se había observado a causa de marcos teóricos estrechos y políticas públicas basadas en la dicotomía urbano/rural), que cuestiona de alguna manera la noción tradicional de modernidad. En la época del consumo, en la era urbana, percibirse identitariamente mixto significa un acto (involuntario, quizás) de contra-racionalidad y de construcción de un camino propio.

El tercer elemento que destacar es la proyección de esta construcción identitaria. Los datos muestran en este sentido tres elementos notables: i) la inexistencia de un tránsito lineal desde adscripciones territoriales subjetivas rurales a urbanas, ii) el movimiento identitario confluye hacia identidades mixtas y iii) este proceso es especialmente fuerte entre los jóvenes. Esto puede estar asociado a mejoras de la conectividad en el interior de la región en análisis y a prácticas de movilidad espacial cotidiana que acortan la distancia simbólica entre lo rural y lo urbano, ligadas al acceso a la educación (CоNCHA, 2013), entre otras prácticas.

Junto con la construcción de la vía mixta (rurbana), sobresale en el análisis de los cambios intergeneracionales el alejamiento de adscripciones rurales; esto posiblemente esté relacionado con la implementación del proyecto agroindustrial y la pérdida de las prácticas productivas y familiares campesinas. En este sentido, aparece acertada la lectura de CANALES y CANALES (2012), que proponen una distinción entre rural y agrario. Si hasta hace algunas décadas ambos conceptos se utilizaban como sinónimos, hoy es evidente que lo agrario necesita un patrón de asentamiento concentrado para poder funcionar (es mucho mejor que la mano de obra para la agroindustria esté concentrada en la ciudad), mientras lo rural es relegado a las formas productivas campesinas. En síntesis, la región es cada vez más agraria y menos rural en términos productivos, y mixta en términos identitarios. 
Se corrobora entonces en términos empíricos lo que teóricamente han propuesto los estudios mencionados en el marco conceptual: el análisis micro-social desestima la teoría de la era urbana o por lo menos la cuestiona en su lógica esencial: un proceso sostenido y homogéneo de expansión de lo urbano, en tanto forma e identidad. Es inevitable interrogarse acerca de las nuevas formas de urbanidad y ruralidad que están emergiendo desde los territorios, más allá de las densidades y tamaños poblacionales. Tanto el concepto de agrópolis como el de rurbanidad pueden aportar en la observación de los nuevos fenómenos identitarios. El primero, ayudándonos a dar cuenta de que la matriz rural es hoy más bien una matriz agraria capitalista y, por tanto, una que tiende a buscar la coexistencia funcional entre ciudad y campo; el segundo, ayudándonos a reconocer el hibridaje cultural urbano-rural que parece constituir hoy en la región del Maule una construcción social relativamente asentada.

\section{Bibliografía}

ÁLVARO, Daniel (2010). “Los conceptos de comunidad y sociedad de Ferdinand Tönnies”, Papeles Del CEIC, 52.

BARBERO, Jesús Martín (1998). "Modernidades y destiempos latinoamericanos”. En Nómadas, 8.

BERDEGUÉ, Julio; JARA, Esteban; MODREGo, Felix; SANCLEMENTE, Ximena; BRENNER, Neil y SCHMID, Christian (2016). "La era urbana en debate". En Eure, 42 (127).

BURDETT, Ricky y RODE, Philipp (2006). “The urban age project”. En Burdett, Ricky y Sudjic, Deyan (Eds.), The endless city, Phaidon, Londres.

CANALES, Manuel y HERNÁNDEZ, María Cristina (2011). "Del fundo al mundo. Cachapoal, un caso de globalización agropolitana”. Espacio Abierto, 20 (4).

CANALES, Manuel y CANALES, Alejandro (2012). "La Nueva Provincia: (re)poblamiento de los territorios agrarios. Chile 1982-2002”. Revista Anales, Séptima Serie, 3.

CIMADEVILLA, Gustavo (2014). "Las formas de la rurbanidad - Anuncios e imágenes". En Congreso ALAIC, Pontificia Universidad Católica del Perú, Lima.

CONCHA, Claudia; ERRÁZURIZ, Tomás; LETELIER, Francisco; MICHELETTI, Stefano; RASSE, Alejandra, y SALCEDO, Rodrigo (2012). “¿Urbano o rural? Repensando territorios, discursos y prácticas al margen de la metrópolis”. En Congreso ALAS, Santiago de Chile.

CONCHA, Claudia (2013). "Trayectorias sociales de sujetos rurales que por primera generación acceden a la educación superior universitaria en la región del Maule, Chile”, Sociedad Hoy, 24.

CONCHA, Claudia y LETELIER, Francisco (2009). "Identidad e Identidades en el Maule, Claves para imaginar el desarrollo regional”. Gobierno Regional del Maule, Talca. 
GALIMBERTI, Silvina (2011). "Rurbanidad, objetos y significaciones. Un estudio acerca de los actores rurbanos y la política pública”. En XXXIV Congresso Brasileiro de Ciências da Comunicação, Recife.

GERMANI, Gino (1963) Política y Sociedad en una Época de Transición. De la sociedad tradicional a la sociedad de masas. Paidós, Buenos Aires.

GÓMEZ, Sergio (2002) La Nueva Ruralidad ¿Qué tan nueva? Editorial Universidad Austral de Chile, Valdivia.

GÓMEZ, Sergio (2003). "Nueva Ruralidad - Fundamentos teóricos y necesidad de avances empíricos”. En Seminario internacional El mundo rural: transformaciones y perspectivas a la luz de la nueva ruralidad, Bogotá.

JAMESON, Fredric (1988). "Cognitive mapping”. En L. Grossberg \& C. Nelson (Eds.), Marxism and the interpretation of culture. Chicago: University of Illinois Press.

KENBEL, Claudia (2006). "A mitad de camino entre lo urbano y lo rural: actores y actividades de rebusque”. UNI revista, 22 (3).

KENBEL, Claudia y CIMADEVILLA, Gustavo (2009). "La rurbanidad desde el enfoque de las memorias sociales”. En X Jornadas Argentinas de Estudios de Población, Asociación de Estudios de Población de la Argentina, San Fernando del Valle de Catamarca.

LETELIER, Francisco, y CONCHA, Claudia (2016). "Nuevas y antiguas identidades regionales: conflicto, exclusión e hibridaje. El caso de la región del Maule”. Eure, 42 (126).

MICHELETTI, Stefano y LETELIER, Francisco (2016). "Aproximaciones al estudio de las prácticas rurbanas en la ciudad intermedia chilena. Actividades de rebusque en Talca”, $B i$ furcaciones, 21.

NISBET, Robert (1967) The Sociological Tradition. Basic Books, Londres.

SOLARI, Aldo (1968) Sociología rural latinoamericana. Paidós, Buenos Aires.

SOROKIN, Pitirim, ZIMERMANN, Carle, y GALPIN, Charles (1930) A Systematic Source Book in Rural Sociology. The University of Minnesota Press, Minneapolis.

UNITED NATIONS POPULATION FUND [UNFPA] (2007) State of world population 2007: unleashing the potential of urban growth. United Nations Population Fund, New York.

UNITED NATIONS HUMAN SETTLEMENT PROGRAMME [UN-HABITAT].(2007). "The state of the world's cities Report 2006/2007-30 years of shaping the Habitat agenda". UN-HABITAT, Londres.

WIRTH, Louis (1938). "Urbanism as a way of life”. En American Journal of Sociology, 44 (1). 